\title{
IL POEMA SACHO DI DANTE ALIGHIERI
}

\author{
Luigi Castagnola \\ (Universidade do Paraná)
}

Nel canto quarfo della Divina Commedia, Dante Alighieri ci racconta come fu condotto da Virgilio a!la presenza degli spiriti magni dell' antichità pagana, radunati in un nobile castello del Limbo. Approssimandosi alla bella scuola dei sommi poeti antichi, Dante vede, non senza compiacente soddisfazione, quattro "grand'ombre" venire incontro a Virgilio ed a lui. Sono quelle di Omero, il

signore dell'altissimo canto

che sopra gli altri com' aquila vola,

di Orazio, di Ovidio e di Lucano.

Dopo che Virgilio ebbe ragionato alquanto insieme con loro, evidentemente per informarli che il suo discepolo sarebbe diventato il maggior poeta italiano, il cui capolavoro l'avrebbe collocato degnamente a fianco dei sommi poeti classici, i quattro grandi salutarono graziosamente Dante e lo accolsero tra loro con onorevole benevoienza.

Da ch'ebber ragionato insieme alquanto, volsersi a me con salutevol cenno;

e 'l mio maestro sorrise di tanto.

$E$ più d'onore ancora assai mi fenno,

ch'ei si mi fecer della loro schiera,

si ch'io fui sesto fra cotanto senno.

Qui, per la prima volta, Dante accenna alla sua missione letteraria e manifesta la certezza del suo glorioso destino di poeta, collocandosi arditamente in compagnia di quella ristretta schiera di poeti la cui autorità godeva nel Medio Evo, e gode tuttavi i. di fama universale. E l'Alighieri divenne, infatti, il maggior poe- 
ta di quella lingua neolatina - chiamata allora, per dileggio, lingua volgare, cioè del volgo - il cui splendido avvenire egli genialmente previde, e di cui fece un'appassionata difesa nel trattato introduttivo del Convivio, dove la nuova lingua delle genti italiche è chiamata "luce nuova, sole nuovo, lo quale sorgerè dove l'usato tramonterà". Perciò scrisse in italiano, e non in latino, la Divina Commedia, il capolavoro poetico, finora insuperato della letteratura italiana, opera veramente sovrana e geniale nella quale "convive l'autorità di una grande coscienza con la voce tumultuosa di tutta un'umanità", libro in cui sempre "cercheremo la testimonianza di ogni grandezza e di ogni bellezza, e pii: della grandezza e della bellezza d'ltalia" (1).

Dante diede al suo poema semplicemente il titolo di Commedia, che Giovanni Boccaccio, più tardi, chiamò divina, aggiunta che fu consacrata poi dall'uso universale dei posteri (2).

Il titolo usato dall' Alighieri aveva un significato alquanto diverso da quello che ha oggi la parola commedia - partico'are genere drammatico. Secondo quanto, sebbene in modo frammentario, ci dice Dante nel De Vulgari Eloquentia e nell'Epistola a Can Grande della Scala, signore di Verona, tre sono gli stili o gradi di eloquenza. Lo stile tragico (o superiore), per i fatti luttuosi, gli alti personaggi, l'amore, le armi e la virtù; il comico (o mezzano), per i fatti ordinari; l'elegiaco (o basso), per le cose tristi (3). Per Dante, quindi, è alta tragedia l'Eneide di Virgilio, per il cárattere eroico della materia e la solennità della poesia, ma non il suo poema, dove, particolarmente nella prima cantica, si tratta di cose basse e volgari e perfino di vizi e tristi passioni. L'Alighieri giustifica, pertanto, il titolo di Commedia, dato alla sua opera, perché essa, come la commedia, ha "principio aspro e fi-

i) Arturo POMPEATI, Storia della Letteratura Italiana, UTET, Torino, 1953, vol. I., p. 506.

2) "Comedia" è, dunque, il vero titolo dell'opera, che Ciovanni Boccaccio, a causa del contenuto religioso e per sentimento d'ammirazione, chiamù "divina"; e con questo nuovo titolo passò ai posteri, quando per la prima volta apparve nell'edizione che Gabriele Giolito pubblicò a Venezia nel 1555." Cf. C. D. LEONI, Introduxione allo Studio della Divina Commedia, Nobel, Săo Paulo, 1951, p. 7.

3) Cf. Dante ALIGHIERI, De Vulgari Eloquentia, II, 4. 
ne felice", e perché "è scritta in stile dimesso (comico, mezza. no), all'opposto dela tragedia, che ha principio mirabile e quieto e fine orribile e stile sublime" (4).

Sono ben conosciute da tutti i dantisti le difficoltà che sorgono per determinare lepoca dela composizione cllla Divina Commedia. Anzitutto è chiaro che un'opera così vasta dovette essere scritta in un periodo di tempo abbastanza lungo. Ciò appare ancor più evidente se si pensa a'la vita agitata, tribolata, raminga e misera dell'esule fiorentino.

La Vita Nuova, composta certamente a Firenze, si chiude col racconto di una visione "ne la quale - afferma Dante — is vidi cose che mi fecero proporre di non dire più di questa benedetta (Beatrice), infino a tanto che io potesse più degnamente trattare di lei. E di venire a ciò io studio quanto posso, si com'ella sae veracemente. Si che, se piacere sarà di colui a cui tutte le cose vivono, che la mia vita duri per alquanti anni, io spero di dicer di !ei quello che mai non fue detto d'alcuna" (5).

E un sicuro annunzio della Divina Commedia. L'idea di questo poema nacque, dunque, neila città del fiore. $E$. se è vero quanto ci narra il Boccaccio, tra le carte della fami . gila Alighieri, salvate nascostamente nel disordine dell'anno fatale (1302) e, più tardi, inviate al poeta esule in Lunigiana, vi erano sette canti dell'Inferno. Non è da escludersi che tali canti siano stati, in seguito, ritoccati o anche rifatti. E corrente la convinzione che la prima cantica, sostanzia!mente, già fosse stata terminata nel 1308. Il Purgatorio fu steso tra il 1309 e il 1313. II Paradiso, scritto certamente negli ultimi anni della vita di Darte, fu pubblicato postumo. Una cosa è certa: quando l'Alighieri viveva a Ravenna era largamente conosciuto come l'autore della Commedia (6).

4) Cosi scrive Dante nell'Epistola a Can Grande della Scala, signore di Verons.

5) Dante ALIGHIERI, Vita Nuova, XLIl.

6) "All'opera intera Dante appose fino dall'origine il titolo, che a noi puj sembrare strano, di "comedia". Ma il medio evo, perduto, per le vicende del teatro nell'età romana, il concetto che il dramma di qualunque forma fosse essenzialmente rappresentazione, soleva dire "tragedie" o "commedie". secondo la natura della materia, i componimenti narrativi." Cf. Vittcrio ROSSI, Storia della Letteratura Italiana, Vallardi, Milano, 1946, $15 a$. ed., vol. I, p. 162. 
Il Poema Sacro di Dante si compone di tre cantiche: L'Inferno, II Purgatorio e II Paradiso. Ognuna di esse abbraccia trentatrè canti, più un canto introduttivo, nell'Inferno. In tuttc, quindi, cento canti. Il verso usato è l'endecasillabo; la strofe è la terzina a rima incatenata, poi chiamata dantesca, perché usata la prima volta dall'Alighieri: aba-bcb-cdc-d...

La concezione astronomica dell' universo, che Dante pose come sfondo cosmico al suo poema, è quella tolemaica. Al centro dell' universo dantesco sta la terra circondata dall'aria; la sfera del fuoco racchiude entro la sua orbita la terra e l'aria. Dante dovrà, quindi, attraversare la regione del fuoco per giun:gere al cielo della Luna. Il cielo dantesco è composto da nove sfere concentriche, sempre più vaste man mano che si allontanano dalla terra. I nove cieli si susseguono in quest'ordine, a partire dalla terra:

1) Cielo della Luna.

2) Cielo di Mercurio.

3) Cielo di Venere.

4) Cielo del Sole.

5) Cielo di Marte.

6) Cielo di Giove.

7) Cielo di Saturno.

8) Cielo Stellato.

9) II Primo Mobile.

Dante, essendosi prefisso di descrivere i tre regni d'oltretomba nel suo poema, si vide costretto a dare, diciamo cosi, una posizione cosmografica ad ognuno di essi. Posizione, naturalmente, fantastica, ma unitaria e solidamente strutturata. L'immaginativa di Dante dimostrò qui una forza creatrice non certo inferiore al volo poetico del suo canto.

L'Inferno fu concepito come avente la forma di un vasto, immenso cono, il cui vertice sta nel centro della terra e l'apertura o base rivo'ta verso l'emisfero settentrionale.

Quando Lucifero fu scacciato dal cielo, precipitò sulla terr.3, nell' emisfero australe, agli antipodi di Gerusalemme, e penetró fino al centro del globo terracqueo. Ivi arrivato il "gran vermo", si fece un ampio vuoto intorno a lui e sopra il suo capo; la terra 


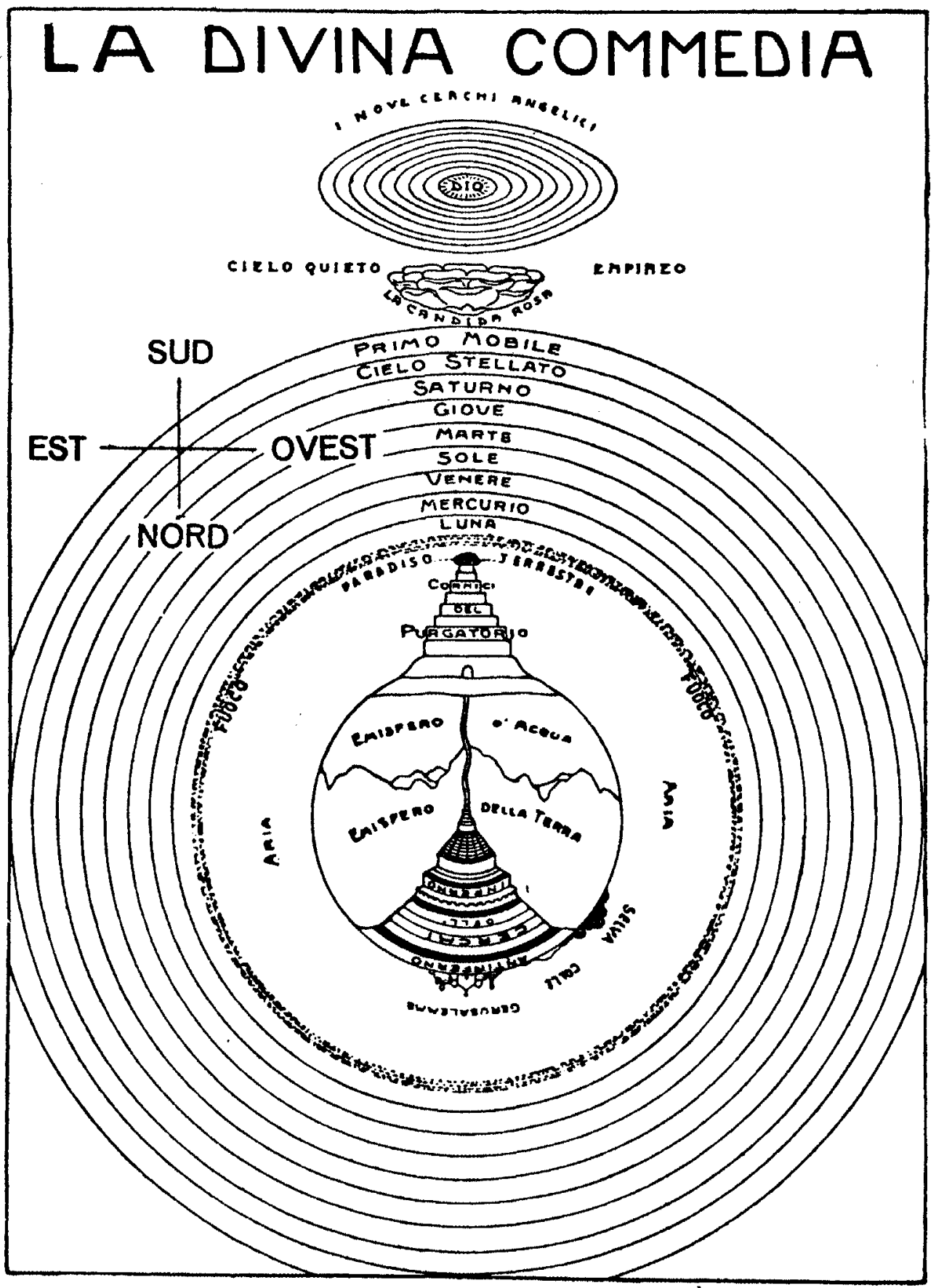

Fig. $N^{\circ} 1$ 


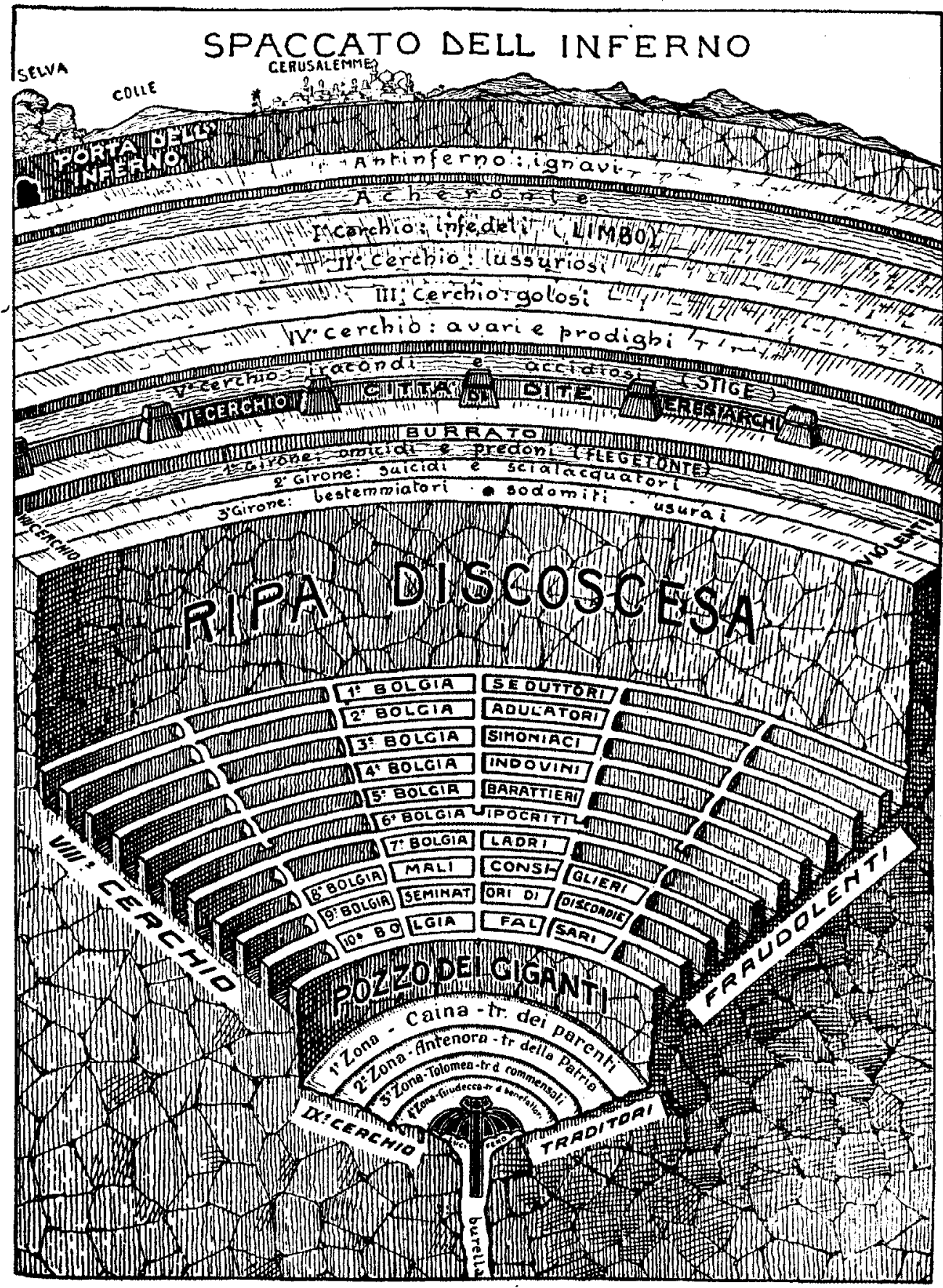

Fig. N. ${ }^{\circ} 2$ 
ritirandosi di li, sali a formare la montagna del Purgatorio, nell'emisfero antartico. Cosi, simultaneamente, ebbero origine il baratro infernale e il colle della purificazione.

La montagna del Purgatorio è circondata dalle acque del mare, poiché le terre emerse dell' emisfero australe, spaventate dall'orribile mostro infernale, Lucifero, là caduto dal cielo, fuggirono verso l'emisfero boreale. Dante, con un "forse", vuol mettere suli" avviso il lettore: non è tutto certo quanto egli dice a rispetto dell' origine del Purgatorio e dell'Inferno. Cosi gli spiegò, per lo meno, Virgilio.

Da questa parte cadde (Lucifero) giù dal cielo;

e la terra, che pria di qua si sporse,

per paura di lui fe' del mar velo,

e venne a l'emisperio nostro; e forse

per fuggir lui lasciò qui 'I luogo vòto

quella ch'appar di qua, e su ricorse.

Dal centro della terra parte la "natural burella" che, attra. versando tutto l'emisfero del sud, porta ai piedi del Purgatoric. Per questo "pertugio tondo" passeranno Dante e Virgilio quan. do saliranno al secondo regno d'oltretomba.

II Paradiso dantesco, o'tre i nove cieli mobili più sopra enumerati, e al di fuori di essi, comprende ancora il cielo quietc, l'Empireo, dove Dante colloca la "candida rosa" della milizia santa, i nove cerchi angelici immoti, stando al centro di tutto il mistero di Dio eterno, uno e trino (7).

\section{L'Inferno (8).}

La struttura dell'Inferno è immaginata dall' Alighieri nel modo seguente.

Antinferno: luogo destinato agl'ignavi.

7) Per avere una vista d'insieme della concezione dell'Universo, qual è descritto e immaginato nella Divina Commedia, si veda la figura n. 1. Dobbiamo alla gentilezza della Casa Editrice Carlo Signorelli, di Milano, tutte le illustrazioni inserite nel presente articolo, prese dalla pubblicazione "Il Poema Sacro", di Andrea Gustarelli. Sono tre fascicoli veramente utilissimi a chi vuole comprendere it poema di Dante.

8) Vedere le figure n. $l$ e n. 2. 


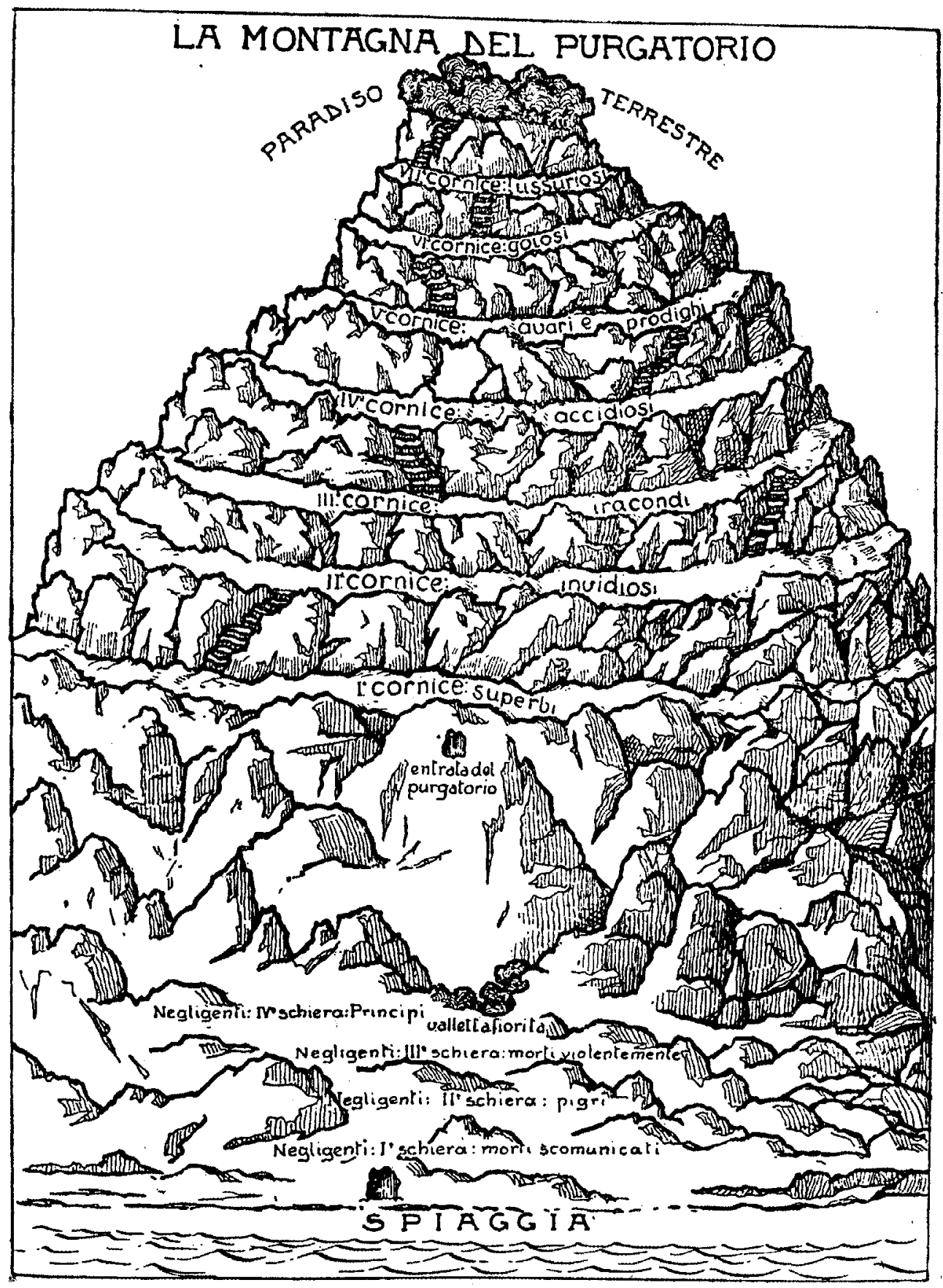

Fig. N. ${ }^{\circ} 3$ 


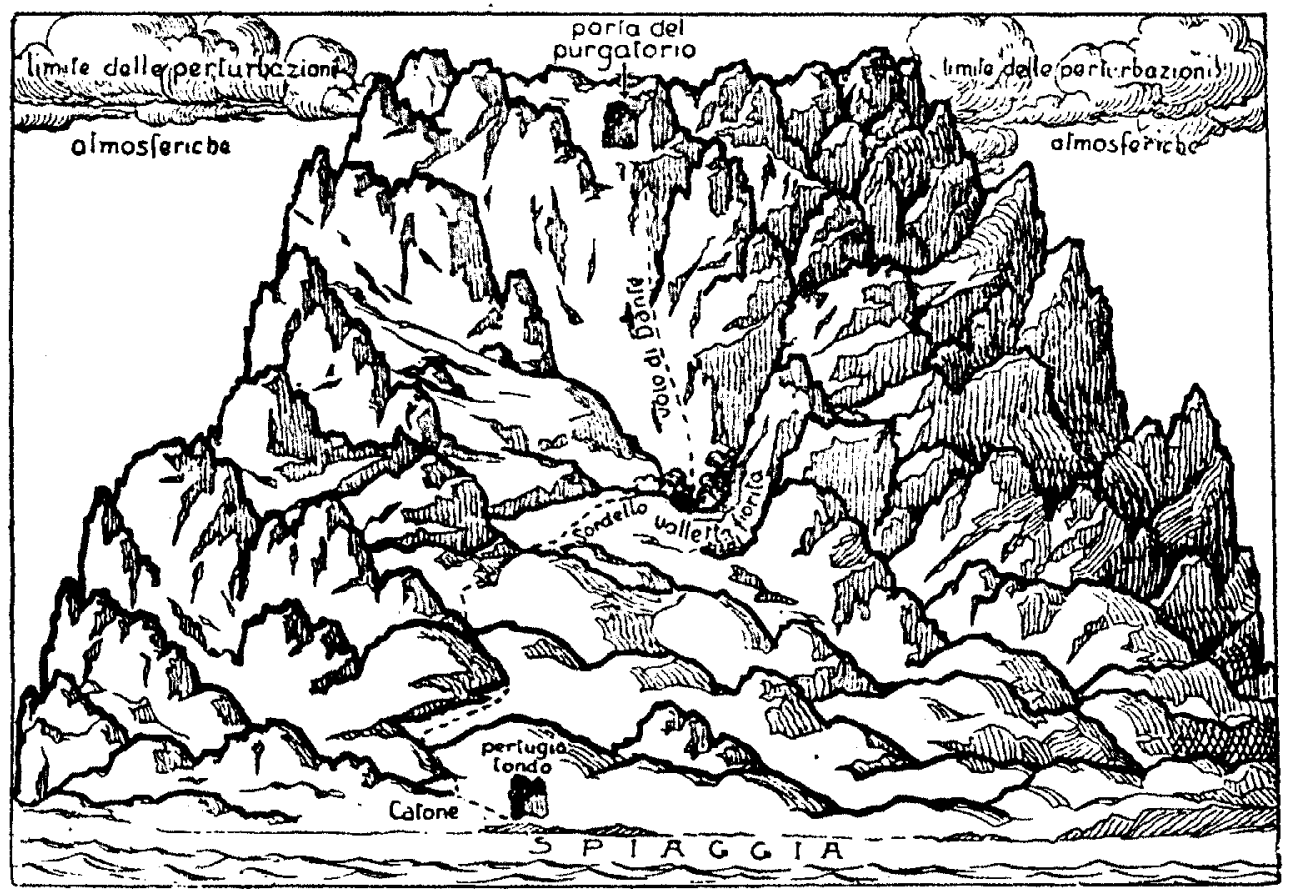

Fig. N. ${ }^{\circ} 4$ 
Acheronte: fiume sotterraneo che cinge circolarmente l'Inferno.

I Cerchio: dimora deg'infedeli (Limbo).

Ii Cerchio: lussuriosi.

III Cerchio: golosi.

IV Cerchio: avari e prodighi.

$\checkmark$ Cerchio: iracondi e accidiosi (palude Stigia).

VI Cerchio: eresiarchi (Città di Dite).

VII Cerchio: violenti. E diviso in tre gironi.

1) Girone: omicidi e predoni (fiume Flegetonte).

2) Girone: suicidi e scialacquatori.

3) Girone: bestemmiatori, sodomiti, usurai.

La ripa scoscesa porta all' ottavo cerchio.

VIII Cerchio: fraudolenti. Questo cerchio è diviso in diec; bolge.

1) Bolgia: seduttori.

2) Bolgia: adulatori.

3) Bolgia: simoniaci.

4) Bolgia: indovini.

5) Bolgia: barattieri.

6) Bolgia: ipocriti.

7) Bolgia: ladri.

8) Bolgia: mali consiglieri.

9) Bolgia: seminatori di discordie.

10) Bolgia: falsari.

Il pozzo dei giganti porta al nono cerchio, chiamato Cocito.

IX Cerchio: traditori. Questo cerchio è diviso in quattro zone.

1) Zona: traditori dei parenti (Caina).

2) Zona: traditori della patria (Antenora).

3) Zona: traditori dei commensali (Tolomea).

4) Zona: traditori dei benefattori (Giudecca).

Infine, al fondo dell'Inferno, si trova Lucifero.

II Purgatorio (9).

La estruttura del Purgatorio è la seguente.

9) Vedere le figure n. 1, n. 3, n. 4. 
A) Antipurgatorio: destinato ai negligenti. Dante li divide in quattro schiere.

1) Schiera: morti scomunicati.

2) Schiera: pigri.

3) Schiera: morti violentemente.

4) Schiera: principi.

B) Purgatorio. Esso comincia là dove è il limite delle perturbazioni atmosferiche, ed è diviso in sette cornici.

1 Cornice: superbi.

II Cornice: invidiosi.

III Cornice: iracondi.

IV Cornice: accidiosi.

$\checkmark$ Cornice: avari e prodighi.

VI Cornice: golosi.

VII Cornice: lussuriosi.

C) Paradiso Terrestre.

II Paradiso (10).

Attraversata la sfera del fuoco, Dante entra nel Paradiso, la cui struttura è la seguente.

I Cielo della Luna: spiriti mancanti.

II Cielo di Mercurio: spiriti operanti.

III Cielo di Venere: spiriti amanti.

IV Cielo del Sole: spiriti sapienti.

$\checkmark$ Cielo di Marte: spiriti militanti.

VI Cielo di Giove: spiriti giudicanti.

VII Cielo di Saturno: spiriti contemplanti.

VIII Cielo delle Stelle fisse: trionfo di Cristo e apoteosi di Maria Vergine.

IX Cielo Cristallino o Primo Mobile: trionfo degli Angeli e della Divinità.

Al di sopra dei nove cieli, che circondano la terra e girano intorno ad essa, si trova immobile l'Empireo. Quivi è la vera e stabile dimora dei beati, radunati nella Candida Rosa. Essi, tuttavia, appaiono a Dante lungo i nove cieli mentovati per dargli un'idea del grado della loro beatitudine.

10) Vedere la figura n. I. 
Nell'Empireo sono collocati, inoltre, i nove cerchi immobili delle gerarchie angeliche al cui centro si trova Dio. Ognuno di questi nove immoti cerchi angelici è motore di uno dei nove cieli sottostanti. A partire da Dio, i nove cerchi angelici si susseguono in quest'ordine.

1) Cerchio dei Serafini (motore del Cielo Cristallino o Primo Mobile).

2) Cerchio dei Cherubini (motore del Cielo delle stelle fisse).

3) Cerchio dei Troni (motore del Cielo di Saturno).

4) Cerchio delle Dominazioni (motore del Cielo di Giove).

5) Cerchio delle Virtù (motore del Cielo di Marte).

6) Cerchio delle Potestà (motore del Cielo del Sole).

7) Cerchio dei Principati (motore del Cielo di Venere).

8) Cerchio degli Arcangeli (motore del Cielo di Mercurio).

9) Cerchio degli Angeii (motore del Cielo della Luna).

Prima di accingerci a seguire il poeta nel suo viaggio oltremondano, sarà conveniente premettere alcune parole per: saperci orientare nel vasto mondo dantesco; conoscere chi furono le sue guide e fin dove esse lo accompagnarono; determinare l'epoca e la durata del lungo viaggio.

Al nord è situata Gerusalemme, sopra la crosta terrestre che copre la grande imboccatura dell'Inferno. Al sud, agli antipodi della città santa, sorge la montagna del Purgatorio, che ha la sua cima diretta verso mezzogiorno. Per chi guarda la figura o tavola N. 1, l'ovest è alla destra e l'est alla sinistra.

Virgilio, che l'Alighieri chiama con affettuosa ammirazione il "suo maestro e il suo autore", accompagna Dante dalla selva selvaggia, in cui s'era smarrito, fino alla sommità del Purgatorio, ed è simbolo della ragione umana in lui personificata. Dal Paradiso Terrestre fino alla Candida Rosa (canto trentunesimo del Paradiso) è accompagnato da Beatrice, simbolo della grazia divina. Dalla Candida Rosa fino al termine del suo neraviglioso viaggio è accompagnato da San Bernardo, simbolo della contemplazione. 
Dante resta nella seiva, disoriantato e spaurito, una notte ed un giorno. Inizia, quindi, il suo viaggio nella notte dell'otto aprile del 1300 — secondo l'opinione di altri, il giorno sette e rimane nel.'Inferno una notte ed un giorno; una notte ed un giorno impiega per salire dal centro dell'Inferno ai piedi della montagna del Purgatorio. All'alba del dieci aprile arriva al Purgatorio, che finisce di visitare a mezzogiono del tredici aprile. Sale alla sfera del fuoco nel pomeriggio del giorno tredici e termina il suo viaggio a mezzanotte del quattordici aprile del 1300 .

Possiamo, ora, fare un breve racconto del Poema Sacro (11).

Prima cantica (L'Inferno).

1. Verso i trentacinque anni, Dante si smarrisce in una selva oscura, simbolo del suo momentaneo traviamento e di quello di tutta l'umanità. Invano tenta il poeta d'uscirne fuori con l'aiuto delle sue forze, insufficienti per trionfare delle passioni. Cli appare Virgilio e lo invita a visitare i tre regni d'oltretomba, essendo questa l'unica via di salvazione che gli resta. La corruzione umana sarà vinta quando verrà sulla terra un misterioso Veltro. Dante accetta l'invito di Virgilio:

Poeta, io ti richeggio

per quello Dio che tu non conoscesti,

acciò ch'io fugga questo male e peggio,

che tu mi meni là dov'or dicesti.

II. Sull'imbrunire, quando tutti gli animali abbandonano ie fatiche del giono e s'avviano al luogo del riposo, Dante s'apparecchia all'arduo cammino. Teme, però, che siano insufficienti le sue forze e dice alla sua guida:

Poeta che mi guidi.

guarda la mia virtù, s'ell' è possente,

prima ch'all'alto passo tu mi fidi.

Virgilio lo rimprovera severamente e, dopo, l'incoraggia dicendogli che si dànno pensiero di lui, nel cielo, la Vergine, santa Lucia e Beatrice. Vinto ogni dubbio, Dante promette che seguirà la sua guida per il cammino alto e silvestro:

11) I numeri romani indicano i canti dell'Infermo. 
Or va, ché un sol volere è d'ambedue:

tu duca, tu signore, e tu maestro.

III. I due poeti attraversano la porta infernale che dura eterna e conduce alla città dolente delle anime dannate. Dante legge al sommo della porta le parole terribili che là sono incise eternamente:

Lasciate ogni speranza, voi ch'entrate.

Virgilio prende per mano il suo discepolo spaventato e l'iritroduce nel vestibolo dell'inferno, dove s'aggirano gl' ignavi.

Quivi sospiri, pianti ed alti guai risonavan per l'aer sanza stelle.

Dante, impressionato da tanto dolore, si mette a piangere, ma Virgilio gli spiega che quei disgraziati non meritano compassione, poiché sono disprezzati tanto dai beati del cielo come dai condannati dell'inferno:

non ragioniam di lor, ma guarda e passa.

Arrivano ad un fiume, l'Acheronte, dove scorgono "venir per nave" il nocchiero Caronte, demonio dagli occhi di bragia, che trasporta i dannati al di là della livida palude con la sua barcaccia. Agli ordini di Virgilio, quel "vecchio bianco per antico pelo" accheta le lanose gote. Un forte terremoto scuote la regione infernale. Dante sviene e quando ritorna in sé già si trova sull' altra riva del fiume, che fu attraversato miracolosamente.

IV. Discendono nel primo cerchio, dove si trova il Limbo, dimora degli spiriti giusti che non ricevettero il battesimo. Quivi, spiega Virgilio a Dante, discese Cesù Cristo per condurre con sé al Paradiso le anime credenti in Cristo venturo. Là, in un no. bile castello, stanno le grandi ombre degli uomini illustri che non conobbero la rivelazione e non entrarono nella chiesa. Virgil:o è uno di essi. Dante è ricevuto con onore da quei savi, che, senza speranza di vedere Dio, vivono "in disio".

$\checkmark$. Con certa nostalgia Dante si congeda dalla savia schicra e discende al secondo cerchio, dove sono puniti $i$ lussuriosi ed è giudice Minosse. Manifesta a Virgilio il desiderio di parlare a due spiriti gentili portati dalla bufera: 
$O$ anime affannate, venite a noi parlar, s'altri nol niega!

Paolo e Francesca da Rimini si avvicinano a Dante "quali colombe, dal disio chiamate". Francesca, con accorata tristezza, racconta fra le lacrime la dolorosa storia di quell'amore che la condusse, col suo amante, al passo fatale. Dante rimane profondamente turbato e cade svenuto per lo strazio sentito davanti alla mestizia dei due sventurati amanti.

VI. I due poeti entrano nel terzo cerchio, dove una pioggia "eterna, maledetta, fredda e greve" sferza i golosi, sopra i quali latra Cerbero, mostro crudele con tre teste. Una di quell'anime interpella Dante. É Ciacco, famoso ghiottone di Firenze, che predice al poeta il suo esilio e le infinite sventure della città del fiore, dilaniata dalle corrotte fazioni politiche.

VII. Con la voce rauca sono salutati sarcasticamente da Pluto nel quarto cerchio:

Papè Satan, Papè Satan, aleppe!...

Parole incomprensibili per Dante, tirato d'imbarazzo dal suo duca savio e gentile, che tutto sapeva. Cli dice Virgilio che sono alla presenza degli avari e dei prodighi. Quindi passa a spiegargli che cosa sia la Fortuna e come essa operi. Scendono, intanto, al quinto cerchio, formato dalla palude stigia, dove sono immerse le anime degl'iracondi e interamente sommerse nel limo quelle degli accidiosi.

VIII. Mentre i poeti attraversano lo Stige sulla barcaccia di Flegiàs, Dante scorge Filippo Argenti, suo nemico. Dice a Virgilio che sarebbe contento di vederlo tuffare in quella "broda". Ed ecco che una turba di dannati si avventano contro Filippo Argenti e fanno strazio di quel fiorentino spirito bizzarro.

Quanti si tengon or lassù gran regi, che qui staranno come porci in brago.

S'avvicinano, frattanto, alla Città di Dite le cui alte mura sono chiuse e ben difese dai diavoli. Virgilio non riesce a convincerli d'aprir loro le porte di Dite. Dante si spaura e vorrebbe tornare indietro, ma riprende animo quando il suo duca gli dice che un messo celeste aprirà foro l'adito della città murata. 
IX. Dopo aver fatto diversi ragionamenti, ecco apparire loro, in cima ad una torre infuocata, le feroci Erinni anguicrinite: Megera, Aletto e Tesifone. Invocano Medusa perché trasformi Dante in pietra. Virgilio protegge attivamente il suo discepolo, e nel frattempo arriva un angelo che rimbrotta $i$ demoni ed apre con autorità le porte delle mura. Entrano nel sesto cerchio dove stanno gli eresiarchi giacenti entro avelii infuocati.

$X-X \mid$. Dante visita le arche di fuoco e s'intrattiene a parlare con alcuni eresiarchi, tra cui Farinata degli Uberti, Cavalcante, padre del poeta Guido Cavalcanti amico dell'Alighieri, Federico II, il re mecenate, fondatore del'a scuola siciliana, ii cardinale Ottaviano degli Ubaldini. Infine Virgilio e Dante si soffermano presso l'avello del papa Anastasio che, secondo l'Alighieri, avrebbe accettato l'eresia di Fotino. Quindi Virgilio spiega a Dante la topografia morale dell'Inferno.

XII - XIII. Per un buratto discosceso discendono al settimo cerchio, dopo aver vinto la violenza bestiale del Minotauro. Il primo girone del cerchio è formato dal Flegetonte, fiume di sangue bollente in cui sono immersi gli omicidi ed $i$ predoni, saettati con frecce dai Centuari. Uno di questi, Chirone, ordina al centauro Nesso di accompagnare i due pellegrini al secondo girone.

II secondo girone è costituito da un bosco strano.

Non fronda verde, ma di color fosco; non rami schietti, ma nodosi e involti; non pomi, v'eran, ma stecchi con tòsco.

Sono quivi puniti i suicidi ed $i$ scialacquatori; i primi sono mutati in sterpi ed $i$ secondi sono inseguiti da cagne bramose che li dilaniano. Su quei pruni nidificano le Arpie, che ululanu sinistre. Dante svelle un ramicello da una pianta e

Come d'un stizzo verde, che arso sia

dail'un de' capi, che dall'altro geme

e cigola per vento che va via,

sì della scheggia rotta usciva insieme

parole e sangue; ond'io lasciai la cima

cadere, e stetti come l'uom che teme.

E Pier della Vigna, cancelliere di Federico II, che parla a 
Dante. Egli fu trasformato in quelio sterpo infernale per essersi suicidato quando l'invidia infiammò contro di lui, fedele al suo signore, gli animi di tutti i cortigiani.

Nel terzo girone, formato da una landa ardente su cui cads una pioggia di fiamme, tribolano bestemmiatori, sodomiti e usurai. Mentre vanno tra quei disgraziati, Virgilio spiega a Dante l'origine dei fiumi infernali. Neli' isola di Creta c'è una montagna, dentro la quale sta il Gran Veglio, ossia una statua colossale rotta da una fessura donde gocciano lacrime che, penetrando poi sotterra, si riuniscono per formare i fiumi dell'Inferno: Acheronte, Stige. Flegetonte e Cocito.

Tra i dannati del terzo girone Dante scorge Brunetto Latini, da lui venerato in vita come un maestro. Con lui s'accompagna e conversa rispettosamente: Brunetto gli predice il suo glorioso destino di poeta:

Se tu segui tua stella, non puoi fallire a glorioso porto.

Salutato pietosamente Brunetto Latini, Virgilio dice a Dante che scenderanno la ripa scoscesa in groppa ad un mostro, Gerione, simbolo della frode. Questa fiera ha la faccia di uomo giusto e il fusto di un serpente dalla coda biforcuta e armata di pungiglioni.

Ecco la fiera con la coda aguzza, che passa i monti e rompe i muri e l'armi ; ecco colei che tutto il mondo appuzza!

XVII - XXX. Dante dedica ben quattordici canti all'ottavo cerchio, diviso in dieci bolge entro cui la infinite schiere dei fraudolenti soffrono le pene più atroci e volgari. Tra i vizi umani, secondo l'Alighieri, comunissimo e ripugnante è quello della frode, eternamente castigato nel vasto Malebolge.

Luogo è, in Inferno, detto Malebolge, tutto di pietra di coior ferrigno come la cerchia che d'intorno il volge.

Nella prima bolgia i seduttori, sconciamente nudi, sono battuti "crudelmente di retro" da demoni cornuti. Nella seconda bolgia Dante dipinge con parole torbide e laide la sconcia pena 
degli adu'atori, tuffati nello sterco (12). Nella terza stanno i simoniaci con la testa fitta entro fori e le piante dei piedi accese fuori delle buche. Nella quarta incontrano gl'indovini distorti orribilmente col capo all'indietro. Nella quinta cuociono nella pece bollente i barattieri, arroncigliati dai demoni. Dante descrive gli sconci procedimenti dei diavoli e le loro zuffe. Nella sesta bolgia vanno con lenti passi gl' ipocriti, oppressi da pesanti cappe di piombo. Tra essi Dante scorse

un, crocifisso in terra con tre pali.

È Caifas che condannò Gesù Cristo.

Attraversato è, nudo, nella via,

come tu vedi; ed è mestier ch'ei senta

qualunque passa, come pesa, pria.

Nella settima boigia i ladri sono avvinti e morsi da serpenti; ridotti a cenere dal morso dei rettili, di nuovo si ricompongono in forma umana. Talvolta sono mutati in serpenti. Prima di lasciare questa bolgia, Dante apostrofa severamente la sua città natale, feconda di ladroni:

Godi, Fiorenza, poi che se' sì grande che per mare e per terra batti l'ali, e per lo 'nferno tuo nome si spande!

12) "In quel nauseante rimescolio d'anime e di feci, Dante scorge un uoms morto da poco e gode nel raccontare d'averlo visto cosi, come gode al pensiero che tanti (chissà quanti n'ha in mente!) di quella razzaccia ora stanno coi capelli asciutti e la bocca piena di miele, ma prima o poi cadranno laggiù, schizzandosi intorno getti d'immondizia come degna raggiera. Non si dica, per carità, che il linguaggio e turpe, che certe cose si potrebbero velare con eufemismi... Gl'ipocriti che arricciano il naso davanti al contenuto della seconda bolgia sono molto affini a coloro che in quella bolgia ingozzano sudiciume. Né si creda che Dante parli per gusto di rovistare nel letame; egli parla per dovere di giustiziere; dall'insopportabile fetore di questa bolgia sale un terribile mćnito agli aduiatori del suo tempo e di tutti i tempi: ma appena può, Dante ritrae lo sguardo e volge altrove il piede: un verso eterno chiude la scena ove non sai se ammirar più la potenza dell'arte o la profondità del senso morale". Cosi, in un bellissimo commento, Dino Provenzal. Cf. Dante ALIGHIERI, La Divina Commedia, commentata da Dino PROVENZAL, Inferno, III ed., Mondadori, Milano, 1949, p. 153. 
Tra li ladron trovai cinque cotali

tuoi cittadini onde mi vien vergogna,

e tu in grande orranza non ne sali.

Nella bolgia ottava vagano i mali consiglieri. Questi frodolenti sono avvolti in fiamme linguiformi. Tra essi sta Ulisse che, pregato da Virgilio, racconta il suo ultimo viaggio alle Colonne d'Ercole e come, oltrepassate quelle, fu inabissato nel." oceano. Nella nona bolgia incontrano nuovi tormenti e nuovi tormentati: i seminatori di discordia. Essi sono feriti orribilmente dalla spada di un demonio. Uno è squartato da cima a fondo e gli penzolano le budella: è Maometto. Finalmente, nella decima bolgia sono tormentati i falsari, sfigurati da malattie ripugnanti, quali la lebbra, la scabbia, l'idropisia e la febbre.

$X X X 1$. La scarsa luce del luogo fa si che Dante, da lontano, scambi per torri i corpi dei giganti che sporgono soltanto per metà fuori del nono cerchio. Sono essi incatenati alie pareti del Pozzo dei Giganti, salvo Anteo che dovrà deporre sul fondo del nono cerchio $i$ due visitatori. Tentarono i giganti dare la scalata al cielo, ed ora sono là impotenti e legati.

$X X X I I-X X X I I I$. I due poeti sono giunti all'ultimo cerchio infernale, detto Cocito. Quivi sono puniti, in quattro zone, i traditori. Nella prima zona - Caina - sono immersi ne! ghiaccio fino al capo i traditori dei parenti. Nella seconda zona - Antenora - i traditori della patria sono immersi nel ghiaccio fino a mezza faccia. Dante incontra fra essi il conte Ugolino che rode eternamente il cranio del suo traditore. Al vedere il poeta fiorentino,

La bocca sollevò dal fiero pasto quel peccator, forbendola ai capelli del capo ch'egli avea diretro guasto;

poi cominciò: "Tu vuoi ch'io rinnovelli disperato do'or che il cor mi preme già pur pensando, pria ch'io ne favelli.

Ma se le mie parole esser den seme che frutti infamia al traditor ch'io rodo, parlare e lagrimar vedrai insieme.

II conte sventurato racconta a Dante l'orrenda morte che 


\section{$-99-$}

i Pisani inflissero a lui ed ai suoi figli innocenti, facendoli morire di fame in una torre (13). Il poeta rimane esterrefatto al sertir narrare l'orrendo misfatto e lascia uscire dalla sua bocca la tremenda apostrofe di condanna contro Pisa:

Ahi Pisa, vituperio delle genti

del bel paese là dove il sì suona,

poi che $i$ vicini a te punir son lenti,

movasi la Capraia e la Gorgona,

e faccian siepe ad Arno in su la foce

si ch'egli annieghi in te ogni persona!

Nella terza zona - Tolomea - i traditori dei commensali sono immersi nel gelo fino alla testa. Dante incontra fra essi Branca d'Oria, genovese, e ne prende l'occasione per fare un'invettiva contro Genova, repubblica marinara rivale di Pisa. In uno stesso canto sono, così, ma'edette le due repubbliche rivali.

Ahi, Genovesi, uomini diversi

d'ogni costume e pien d'ogni magagna, perché non siete voi del mondo spersi?

$X X X I V$. Al centro della quarta zona - Giudecca - e dell'Inferno sta l'imperatore del doloroso regno. In questa zona sono puniti $i$ traditori dei benefattori, totalmente immersi nel ghiaccio. Lucifero è un mostro così orrendo che Dante sente difficoltà a descriverlo. Sporge fuori della ghiaccia da mezzo il petto, e con le sue ali di pipistrello immondo produce un vento cosi freddo che fa congelare tutto Cocito. Da lui procede ogni

13) "Dante ha ascoltato il racconto senza batter ciglio... ma egli ha ormaiil cuore impietrito: non sente più pietà per i traditori. Finalmente un grido gli esce dal labbro e dall'anima, ma non di pietà: di esecrazione. In quest'atmosfera d'odio, anche Dante non ha che una passione: l'odio... Virgilio, lì presente, non aggiunge una parola né per approvare né per biasimare: quando i mezzi datigli da Dio l'uomo adopera per infuriare contro il prossimo, per confondere, in una sola rabbia di distruzione, innocenti e colpevoli, la ragione non ha più nulla da dire: siamo fuori del suo dominio... Episodio dei più grandi del poema, anzi uno dei più meravigliosi che abbia mai prodotti la letteratura d'ogni paese". Cf. Dino PROVENZAL, op. cit., p. 291. 
male: alla presenza di questo mostro Dante, dallo spavento, rimane come chi non è vivo nè morto. Aveva tre facce e tre bocche.

Con sei occhi piangeva, e per tre menti gocciava it pianto e sanguinosa bava.

Da ogni bocca dirompea co' denti

un peccatore, a guisa di maciulla,

si che tre ne facea cosi dolenti.

Lucifero maciulla entro le sue bocche feroci Giuda, traditore di Gesù Cristo, Bruto e Cassio, traditori di Giulio Cesare.

Ormai Virgilio ha mostrato al suo discepolo tutto il male dell'Inferno. Scendono ora lungo il corpo di Lucifero, fino alle anche; quivi, con difficoltà, si capovo'gono. Si trovano già nell'emisfero australe in uno spazio circolare corrispondente alla parte opposta di Cocito. Virgilio spiega a Dante l'origine dell'Inferno e gi i schiarisce altri dubbi. Quindi, salendo per una "burella" buia, s'incamminano verso il chiaro mondo australe, e là arrivando rivedono con somma gioia le stelle.

Seconda cantica (II Purgatorio) (14).

I. I due poeti, dopo aver lasciato l'Inferno, sentono un diletto profondo nel contemplare un mondo più dolce e sereno. Il bel pianeta di Venere fa ridere tutto l'oriente, il cielo del mattino ha un dolce colore di zaffiro. Brillano in alto

\section{...... quattro stelle}

non viste mai fuor che alla prima gente.

E la costeliazione della Croce del Sud, che illumina il volto di Catone, custode del regno del Purgatorio. I due poeti s'intrattengono affabilmente con quel saggio degno di riverenza. Virgilio gli dice che il suo discepolo non vide ancora "l'ultima sera" la morte - e fa quel viaggio per volontà divina.

Or ti piaccia gradir la sua venuta:

libertà va cercando, ch'è sì cara, come sa chi per lei vita rifiuta.

Allontanatosi l'Uticense, Virgilio sottopone Dante ad un 
significativo atto d'umiltà e quindi entrambi si muovono verse, la spiaggia.

11. Sul mare, da lungi, risplende un fuoco. È il prime angelo che s'avvicina, conducendo per nave più di cento spiriti destinati al Purgatorio. Depostili sulla spiaggia, l'angelo ritorna a prenderne altri presso la foce del Tevere. I nuovi venuti chiedono ai nostri due pellegrini dove sia il cammino della montagna, ma questi dichiarano d'essere stranieri in quel luogo. Dante. meravigliato, scorge tra quelle anime salve il suo amico Casella, musico fiorentino, che canta alcune canzoni dantesche. E tale la dolcezza di quel canto che tutti dimenticano di continuare il viaggio, finchè Catone li rimprovera. Come colombi improvvisamente spaventati, gli spiriti si sbandano velocemente. Né la "nostra partita fu men tosta", aggiunge il poeta.

III. Avviandosi verso la montagna, incontrano la prima schiera dei negligenti. Virgilio, nel frattempo, aveva spiegato al suo discepolo che non si possono comprendere i misteri di Dio.

Matto è chi spera che nostra ragione possa trascorrer la infinita via che tiene una sustanza in tre persone.

State contenti, umana gente, al quia; ché, se potuto aveste veder tutto, mestier non era partorir Maria.

Virgilio chiede a quegli spiriti eietti che indichino loro la strada. E questi, come fanno le pecorelle che escono dall'ovile, timidi si approssimano e additano il cammino. Uno di loro parla a Dante:

biondo era e bello e di gentile aspetto, ma l'un de' cigli un colpo avea diviso.

Era Manfredi, figlio di Federico II. Gli racconta come avvenne la sua morte e gli dice che, colpito da due punte mortali, si arrese piangendo, a Quei che volentier perdona.

IV. Ripreso il cammino, sempre conversando e sempre affrontando le difficoltà dell'erta dirupata, talvolta sostando per contemp'are e riposarsi, Dante è apostrofato ironicamente dalla voce di uno spirito cosi negligente nel comportamento che la pi- 
grizia pareva "sua sirocchia". Era Belacqua, fiorentino amico di Dante, ora là tra la seconda schiera dei negligenti dell'Antipurgatorio. L'antica amicizia tra Dante e Belacqua permette a questi di parlare al poeta con accenti confidenzili di scanzonatura.

V-VI. Riprendendo il cammino, Dante è distratto dalla curiosità di vedere spiriti che additano verso di lui, meravigíati che il suo corpo proietti ombra sul terreno. Virgilio lo rimprovera dell'indugio:

Vien dietro a me, e lascia dir le genti!

Sta come torre ferma, che non crolla

giammai la cima per soffiar de' venti.

Incontrano una terza schiera di negligenti "per forza morti". Tra essi vede Buonconte da Montefeltro, ed ascolta l'accorata supplica di una gentile voce femminile, quella di Pia dei Tolomei:

Deh, quando tu sarai tornato al mondo,

e riposato della lunga via...

ricòrditi di me, che son la Pia!

Virgilio si dirige verso un'anima solitaria per sapere da essa il miglior cammino. Era un lombardo, anzi, un mantovano come Virgilio, il trovatore Sordel'o. L'amor patrio si manifestò inten. so e sincero tra i due, che si abbracciarono. Dante ne approfitta per lanciare un'invettiva amara contro le genti d'ltalia sempre in guerra tra loro, sebbene tutte italiane.

VII - VIII. Abbracciato Virgilio più volte, Sordello accompagna i due poeti verso la valletta fiorita, dimora dei principi.

Era già l'ora che volge il disio

ai naviganti e intenerisce il core, lo di ch'han detto a' dolci amici addio;

e che lo novo peregrin d'amore

punge, se ode squilla di lontano

che paia il giorno pianger che si more.

Quelle anime recitano dolcemente la loro prece serotina: Te lucis ente. Vedono, quindi, due biondi angeli scendere dal 
cielo per difendere le anime delía valletta amena contro il serpente tentatore,

forse qual diede ad Eva il cibo amaro (15).

IX. Dante s'addormenta nella valletta fiorita ed ha un sogno. Gli pare che un'aquila lo ghermisca e lo trasporti nella ragione del fuoco. Svegliato di soprassalto, Virgilio gli dice che Lucia, durante il sonno, discese e la trasportò davanti alla porta del Purgatorio. Sordello rimase nell'Antipurgatorio.

Un angelo è posto a custodia della porta del Purgatorio, alia quale si accede attraversando tre gradini. Dante chiede umilmente all'angelo portiere che gli conceda entrare.

Devoto mi gittai a' santi piedi;

misericordia chiesi che m'aprisse, ma pria nel petto tre fiate mi diedi.

Sette $P$ nella fronte mi descrisse col punton della spada, e: "Fa che lavi, quando se' dentro, queste piaghe!" disse.

Aperta la porta, Dante entra con Virgilio, e ode il canto de! Te Deum laudamus.

$X-X \mid 1$. I nostri pellegrini sono giunti alla prima cornice del Purgatorio, dove $i$ superbi si muovono lentamente caricati $d i$ massi pesanti. Vedono scolpiti sulla ripa marmorea esempi d'umiltà con un'arte superiore a quella di Policleto. Dante, alla vista della triste ed umiliante pena, fa un energico ammonimento agli orgogiiosi di tutti $i$ tempi.

O superbi cristian, miseri lassi

che, della vista della mente infermi,

fidanza avete ne' ritrosi passi,

non v'accorgete voi che noi siam vermi

nati a formar l'angelica farfalla

che vola alla giustizia sanza schermi?

Recitando devotamente il Pater noster, queile anime chie-

15) Forse lo stesso serpente che sedusse Eva. 
dono perdono del loro orgoglio folle. La gloria umana è un soffio di vento che sparisce.

Credette Cimabue nella pittura

tener lo campo, e ora ha Giotto il grido,

si che la fama di colui oscura.

Cosi ha tolto i'uno all'altro Guido

la gloria della lingua; e forse è nato

chi l'uno e l'altro caccerà del nido.

L'angelo dell'umiltà scance'la dalla fronte di Dante il primo $P$, e quindi invita $i$ due poeti a salire alla seconda cornice.

$X I I I-X V$. Ivi sono gl'invidiosi. Indossano il cilicio ed hanno gli occhi cuciti con fili di ferro. Per l'aria si espondono voci che ricordano esempi di carità. Dante incontra là molti personaggi illustri e parla con alcuni di essi. L'angelo della misericordia scancella dalla fronte del poeta la seconda $P$ e Dante s'accorge che sono giunti alla terza cornice.

$X V I$ - XVII. In una densa nube di fumo vanno gl'iracondi, cantando l'Agnus Dei e contemplando esempi di mansuetudine. Marco Lombardo spiega a Dante che non si deve attribuire all'influsso degli astri, come ta'uni stoltamente credono, ogni fatto umano. Se cosi fosse, sarebbe distrutto il libero arbitrio e sarebbe ingiusto punire chi fa il male e premiare chi opera il bene.

L'angeio della pace scancella la terza $P$ dalla fronte di Dante e invita i due poeti a salire alla quarta cornice. Quivi giunti, Virgilio spiega al suo discepolo un'ingegnosa teoria dell'amore. nonchè la topografia morale del Purgatorio.

XVIII - XIX. Nella quarta cornice, dimora degli accidiosi, Virgiiio continua le sue spiegazioni filosofiche, fino a che Dante vede una schiera di anime che corrono velocemente, udendo esempi di sollecitudine glorificata e di accidia punita, gridati dagli stessi accidiosi. Finalmente Dante si addormenta ed ha un sogno, che Virgilio gli spiegherà dopo che si sarà destato.

L'angelo della sollecitudine toglie ia quarta $P$ dalla fronte di Dante, e dopo avviene la salita alla quinta cornice. Sul pavimento giacciono bocconi gli avari e i prodighi con mani e pied: legati. 


\section{$-105-$}

XX-XXIII. Mentre camminano sulia quinta cornice, i due poeti odono un terremoto e quindi da ogni parte s'alza it canto del Cloria in excelsis Deo. Tra le persone là incontrate è il celebre poeta latino Stazio, che fa un appassionato elogio di Virgilio, senza sapere d'averlo davanti. Dante g'íielo dice e Stazio vorrebbe inchinarsi ad abbracciare i piedi del suo maestro, ma questi glielo vieta. Stazio, che nella Tebaide si rivela ancora pagano, spiega a Virgilio come si fece cristiano. Liberato ora dalle. pene del Purgatorio, si unisce ai due poeti e con loro sale alla sesta cornice, dopo che Dante fu liberato dalla quinta P dall'angelo della giustizia.

Virgilio è seguito da Stazio e questi da Dante. Cammin facendo nella sesta cornice, incontrano i golosi che soffrono la fame e la sete, acuite dalla vista di alberi carichi di frutti saporiti e da un'acqua che scende limpidissima dalla roccia.

XXIV - XXVI. Dante parla con Forese Donati, suo amicc, e con Bonagiunta Orbicciani. Questi gli chiede se è veramente quel Dante che diede origine ad una nuova forma poetica scrivendo la famosa canzone" Donne ch'avete intelietto d'amore". L'Alighieri risponde con i celebri versi:

$$
\begin{aligned}
& \text {...... lo mi son un che, quando } \\
& \text { amor mi spira, noto, e a quel modo } \\
& \text { ch'ei ditta dentro vo significando. }
\end{aligned}
$$

Liberato Dante dal'iangelo dell'astinenza dalla sesta $P, i$ tre poeti salgono sulla settima cornice, dove $i$ lussuriosi camminano tra le fiamme. Stazio spiega la teoria della generazione dei cor$\mathrm{pi}$, in cui Dio infonde l'anima razionale. Tra i lussuriosi Dante incontra Guido Guinizelli, l'iniziatore della famosa scuola degli stilnovisti, e lo chiama padre

mio e degli altri miei miglior che mai

rime d'amore usàr dolci e leggiadre.

$X X V I I-X X X$. Non senza timore, Dante attraversa le fiamme delia cornice, e quando si trova al di là del fuoco anche la settima $\mathrm{P}$ è scomparsa dalla sua fronte, scancellata dall'angelo della castità o dal fuoco. I tre poeti passano la notte dormendo seduti su tre gradini. Al mattino Virgilio annunzia al suo disce. 
polo che arriveranno tra poco al Paradiso Terrestre e quindi si congeda da lui, poichè la sua missione è finita.

Tratto tho qui con ingegno e con arte;

lo tuo piacere omai prendi per duce;

fuor se' dell'erte vie, fuor se' dell'arte.

Non aspettar mio dir più nè mio cenno:

libero, dritto e sano è tuo arbitrio.

e fallo fòra non fare a suo senno;

perch'io te sopra te corono e mitrio.

Dante passeggia 'entamente tra i fiori e gli alberi del Paradiso Terrestre, fino a che gli appare, al di là del fiume Letè, Matelda dagli occhi splendenti come quelli di Venere. Matelda spiega a Dante molte cose relative al luogo ove si trovano e gli dice che l'acqua del Letè scancella la memoria del peccato e quella dell'Eunoè ravviva il ricordo del bene fatto. Continuando il cammino, Matelda su d'una riva del fiume e Dante sull'altra, incontrano una mistica processione in mezzo alla quale un carro è tirato da un Grifone. In seguito, tra una nuvola di fiori, gli appare Beatrice "vestita di color di fiamma viva". Dante, confuso e tramortito, si volge per par'are a Virgilio, ma s'accorge che "il dolcissimo padre" era sparito e non può frenare il pianto. Beatrice rimprovera a Dante i suoi traviamenti giovanili.

$X X X I-X X X \mid I I$ Finalmente il poeta si pente di quel che ha fatto e si confessa. Poi cade a terra svenuto pel rimorso. Quando rinviene è immerso nelle acque del Letè che attraversa aiutato da Matelda e, dopo aver inghiottito un poco di acqua, dimentica le passate brutture. Allora Beatrice gli si svela nella sua sublime bellezza.

La processione riprende il cammino, e Dante e Stazio vanno a lato del carro che subisce varie trasformazioni. Beatrice va spiegando molte cose a Dante, che però non riesce a compren. dere pienamente, specialmente quando essa parla, con linguaggio profetico, di un duce che ridarà onore ail'autorità imperiale (16).

16) Le lettere $D \times V$, anteponendo il $V$ al $X$, dànno $D V X$, duce, $e$, in numeri romani, significano: cinquecento, dieci e cinque. 
Per ordine di Beatrice, Matelda fa bere a Dante l'acqua del fiume Eunoè, ed egli sente allora ravvivato il ricordo del bene operato. Pienamente purificato. Dante ha terminato la visita al Purgatorio ed è pronto per salire al cielo.

lo ritornai dal'a santissimionda rifatto sì come piante novelle rinnovellate di novella fronda, puro e disposto a salire alle stelle.

Terza cantica (II Paradiso) (17).

I - IV. Dante, con tre famose terzine, annuncia la diffico'tà che prova nell'esporre poeticamente la materia della terza cantica.

La gloria di Colui che tutto muove per l'universo penetra e risplende in una parte più e meno altrove.

Nel ciel che più della sua luce prende fu' io, e vidi cose che ridire né sa né può chi di lassù discende; perché appressando sé al suo disire, nostro intellettó si profonda tanto, che dietro la memoria non può ire.

Ascende con Beatrice alla sfera del fuoco, mentre ascolta le profonde lezioni che gli fa la sua nuova guida. Chi non ha fio: d'ingegno torni indietro e non lo segua nel suo racconto, poichè non comprenderebbe nulla. Salito al cielo della Luna, incontra ivi le anime che mancarono ai voti di castità; esse gli sembrano immagini riflesse in vetri 0 in acque cristalline. Beatrice va snebbiando la mente di Dante da vari dubbi astronomici, teologici e morali.

V - VII. Rapidi come una saetta, Beatrice e Dante salgono al cielo di Mercurio, patria beata degli spiriti attivi. Questi appaiono a Dante come tanti splendori sfavillanti e danzanti. Uno di quegli spiriti, interpellato dal poeta, dice d'essere Giustiniano imperatore. Fa un'appassionata e polemica storia dell'aquila roma-

17) I numeri romani indicano i canti del Paradiso. 
na, simbolo de'l'Impero, grandioso sogno di Dante. L'aquila romana operò cose eccezionali quando era recata da Giulio Cesare.

Quel che fe' poi ch'egli usci di Ravenna

e saltò Rubicon, fu di tal volo.

che nol seguiteria lingua né penna.

Il poeta lancia invettive contro le fazioni dei Cuelfi e dei Ghibellini, nemici della vera aquila imperiale, alla cui autorità $s_{4}$ debbono inchinare tutti $i$ popoli.

Beatrice continua ad illuminare Dante, spiegandogli tutti i dubbi e tutte le questioni che assalgono la sua mente.

VIII - IX. Per lo cresciuto splendore di Beatrice. Dante s'accorge d'essere già dentro il cielo di Venere, dimora degli spiriti amanti. Essi girano intorno cantando. Uno di loro, Carlo Martello, parla a Dante e gli spiega perchè i figli molte volte sono diversi dai padri. Altra facella che s'intrattiene a dialogare con Dante è quella di Cunizza da Romano, e questi addita Folco da Marsiglia, che apostrofa Firenze e l'avarizia degli ecclesiastici.

$X$-XIV. Entrato nel cielo del So'e, v'incontra le anime dei sapienti, disposti in tre corone di fulgori che girano cantando. Dante invita il lettore a contemplare la mirabile architettura celeste, che egli va spiegando come un maestro al discepolo.

Messo tho innanzi: omai per te ti ciba;

ché a sé torce tutta la mia cura

queila materia ond'io son fatto scriba.

Tommaso d'Aquino addita al poeta gli spiriti sapienti della corona beata, che, in seguito, riprende il suo movimento di danza ed il suo canto.

Indi, come orologio che ne chiami

nell'ora che la sposa di Dio surge

a mattinar lo sposo perché l'ami,

che l'una parte l'aitra tira e urge,

tin tin sonando con sì dolce nota, che 'I ben disposto spirto d'amor turge;

cosi vid'io la gloriosa rota

muoversi e render voce a voce in tempra

ed in dolcezza ch'esser non può nota

se non colà dove gioir s'insempra. 
È tanta la felicità di Dante, là nel cielo del So'e, che sente la necessità di compatire e disprezzare gli uomini stoltamente presi dalle cure insensate di questo mondo. Povera umanità che $s^{\prime}$ invesca in raggiri giuridici, in ruberie, in lascivi amori, in ogni specie di violenza, o s'abbandona ali'ozio:

$$
\begin{aligned}
& \text { quanto son difettivi sillogismi } \\
& \text { quei che ti fanno in basso batter l'ali! }
\end{aligned}
$$

Tommaso d'Aquino fa, quindi, l'elogio di San Francesco, l'innamorato della povertà e della natura. Un'altra corona di anime fuigenti vede Dante; tra esse è San Bonaventura che fa l'elogio di San Domenico e addita al poeta gli spiriti sapienti che stanno con lui. Riprende la parola San Tommaso, e schiarisce alcuni dubbi di Dante. Dopo di che il poeta ammonisce gli uomini a non affrettare $i$ loro giudizi sul prossimo, poichè egli vide già il pruno senza foglie durante l'inverno, ma in primavera

$$
\text { ...... portar la rosa in su la cima. }
$$

Nuovi dubbi di Dante sono sciolti da Salomone. Dopo aver contemplato una terza corona di spiriti sapienti, sale con Beatrice al cielo di Marte.

XV - XVII. Quivi gli spiriti lucenti di coloro che lottarono e morirono per difendere la Fede si manifestano a Dante come splendori rosseggianti. Anch'essi si muovono e cantano, formando una croce in cui lampeggia Cristo.

Uno di quegli spiriti, simi.e ad un "vivo topazio", saluta Dante con effusione giubilante, riconoscendo in lui uno dei suoi discendenti:

O fronda mia in che io compiacemmi pure aspettando, io fui la tua radice.

E Cacciaguida, trisavolo del poeta; questi rimane così con. tento di sapere che la sua famiglia discende da Cacciaguida, in cielo spirito militante $e$ in terra soldato crociato, che con lui s'intrattiene lungamente, chiedendo notizie su Firenze antica, sui suoi antenati, e sollecitando spiegazioni circa il suo futuro. Cac ciaguida predice chiaramente al poeta il suo esilio: 


\section{$-110-$}

Tu proverai si come sa di sale

lo pane altrui, e come è duro calle

lo scendere e 'I salir per l'altrui scale.

E quel che più ti graverà le spalle,

sarà la compagnia malvagia e scempia

con la qual tu cadrai in questa valle.

II glorioso antenato di Dante riprende, in seguito, a parlare per indicare al pronipote varie anime beate che si trovano nel cielo di Marte. Riguardando Beatrice, Dante la vede più lucente. e da ciò s'accorge d'essere già salito al cielo di Giove.

$X I X-X X$. Nel sesto cielo si rivelano a Dante gli spiriti giusti. Essi cantano e si dispongono in forma di lettere e quind formano un'aquila che parla al poeta, dicendogli, tra le altre cose, che la giustizia divina è tanto alta che gli uomini frequente mente non la intendono.

\section{Quali}

son le mie note a te. che non le 'ntendi, tal è il giudicio eterno a voi mortali.

L'aquila spiega a Dante i suoi dubbi intorno alla predestinazione e addita alcuni degli spiriti giusti più illustri, come Davide, Traiano, Costantino ed altri ancora.

XXI - XXII. Beatrice parla a Dante e g'i dice che non può aumentare il suo splendore poichè egli non lo potrebbe sopportare tanto è grande. Sono già nel settimo cielo, dove gli spiriti contemplanti splendidissimi salgono e scendono i gradini aurei di una scala immensa di cui non si vede la sommità. Nel cielo di Saturno il lume di San Pier Damiano parla a Dante per sciogliere alcune sue incertezze e per apostrofare il lusso degli ecclesiastici. A sua volta San Benedetto, che in vetta a Montecassino portò

lo nome di Co'ui che in terra addusse

la verità che tanto ci sublima,

condanna la corruzione dei monaci. Dante sale quindi rapidiss! mamente la scala santa ed arriva con Beatrice al cielo delle stelle fisse, donde contempla la terra, "l'aiuola che ci fa tanto feroci". 
XXIII - XXVII. Nell'ottavo cielo migliaia di spiriti trionfanti appaiono a Dante in forma di lucerne illuminate dal Sole di Cristo. Vede il trionfo del Figlio di Dio è l'apoteosi di Maria Vergine. San Pietro interroga Dante sulla Fede e il poeta risponde facendo la sua solenne professione di fede:

$$
\begin{gathered}
\ldots \ldots \ldots \text {. } . \ldots \text { credo in uno Dio } \\
\text { solo ed eterno che tutto il ciel move } \\
\text { non moto } \ldots \ldots \ldots \ldots \ldots \ldots \\
\text { credo in tre Persone eterne, e queste } \\
\text { credo una Essenza si una e si trina, } \\
\text { che soffera congiunto sono ed este. }
\end{gathered}
$$

San Jacopo esamina il poeta intorno alla Speranza e San Giovanni evangelista lo interroga sulla Carità. Adamo, poi, appare al poeta e gli parla del suo peccato. Sale, quindi, al nono cielo, dove le gerarchie angeliche volteggiano attorno ad un punto luminoso: Dio.

XXVIII - XXIX. Nel cielo Cristallino, o Primo Mobile, Beatrice spiega al suo protetto il meccanismo dei cieli e lo illumina intorno ai nove cerchi angelici, motori dei nove cieli che egli ha visitato, e circa la ribellione degli angeli cattivi.

XXX - XXXIII. Scomparsi i cori angelici, Beatrice appare a Dante d'una bellezza indescrivibile. Essi sono giunti al cielo Empireo,

$$
\text { ch'è pura luce: }
$$

luce intellettual, piena d'amore.

Nell'Empireo, patria degli Angeli, dei Beati e di Dio, Dante vede una fiumana di luce corruscante, da cui escono faville che si vanno a posare sui fiori delle rive per poi ritornare a tuffarsi nei fiume di luce.

E vidi lume in forma di rivera

fulvido di fulgore, intra due rive

dipinte di mirabil primavera.

Di tal fiamma uscian faville vive,

e d'ogni parte si mettean nei fiori,

quasi rubin che oro circonscrive;

poi, come inebriate dagli odori, riprofondavan sé nel miro gurge. 
In seguito la fiumana lucente prende forma di Candida Rosa, e Beatrice conduce Dante attraverso la Candida Rosa, verso it suo centro. Il poeta guarda stupito da ogni parte e si volge a Beatrice per interrogarla. Ma essa è sparita per lasciare in suo luogo. come guida, San Bernardo. Questi rivolge la sua parola a Dante e l'accompagna alla visione della Vergine, alla cui presenza arrivati, il santo vecchio, con mirabile preghiera, le chiede che faccia vedere al poeta la gloria di Dio.

Vergine madre, figlia del tuo Figiio, umile ed alta più che creatura, termine fisso d'eterno consiglio...

Qui se' a noi meridiana face

di caritate; e giuso, intra i mortali,

se' di speranza fontana vivace.

Donna, se' tanto grande e tanto vali,

che qual vuol grazia ed a te non ricorre,

sua disianza vuol volar sanz'ali...

Or questi .............

Supplica a te, per grazia, di virtute

tanto, che possa con gli occhi levarsi

più alto verso l'ultima salute.

La preghiera di San Bernardo è esaudita e Dante ha la visione di Dio, uno e trino. II poeta vorrebbe descrivere l'Essere Supremo, ma sente di poter balbettare soltanto qualche parola e preferisce rinunciare all'impresa, conscio che la sua poesia, per quanto alta e sublime, è assolutamente incapace di tanto:

All'alta fantasia qui mancò possa.

Arrivati alla fine di questo viaggio, meraviglioso e terribile allo stesso tempo, un senso di stupore ci assaie. Stupore per l'immensità del genio poetico che l'ideò e creò, stupore per il fine grandioso che Dante si prefisse di raggiungere.

La lingua italiana Dante la trovò formata, ma gracile ancora e assai timida. Egli la seppe piegare e dominare con padronanza dispotica si da renderla strumento atto ad sprimere ogni concetto, a scolpire ogni immagine, e usci dalia dura prova trionfalmente vittorioso. 
La Divina Commedia, secondo l'intenzione dell'Alighieri, vuol essere un tentativo energico, fiducioso, caritatevole, titanico, umano e cristiano di trasformare l'umanità. Dante volle tentare, mediante l'arte, di cambiare lo stato dell'uomo sulla terra, elevandolo dalla miseria brutale alla felicità della giustizia, dalla vita infernale di questo mondo alla beatitudine di una città paradisiaca. Come disse uno dei più innamorati ammiratori del poema sacro, la Commedia "è un miracolo di poesia che voleva operare un effettivo miracolo spirituale e perciò non appartiene soitanto alla piccola storia della letteratura ma, prima di tutto, alla storia misteriosa e dolorosa del genere umano" (18).

18) Giovanni PAPINI, Dante Vivo, L. E. F., Firenze, 1933, p. 366. 